\title{
Sharpening the particle knife: the evolution of ultra-precise proton therapy
}

\author{
J. Isabelle Choi ${ }^{1,2}$, Shaakir Hasan ${ }^{1}$, Robert H. Press ${ }^{1}$, Arpit M. Chhabra ${ }^{1}$, Charles B. Simone II ${ }^{1,2}$ \\ ${ }^{1}$ Department of Radiation Oncology, New York Proton Center, New York, NY, USA; ${ }^{2}$ Department of Radiation Oncology, Memorial Sloan \\ Kettering Cancer Center, New York, NY, USA \\ Contributions: (I) Conception and design: JI Choi, CB Simone 2nd; (II) Administrative support: JI Choi, CB Simone 2nd; (III) Provision of study \\ material or patients: JI Choi; (IV) Collection and assembly of data: JI Choi; (V) Data analysis and interpretation: All authors; (VI) Manuscript writing: \\ All authors; (VII) Final approval of manuscript: All authors. \\ Correspondence to: J. Isabelle Choi, MD. Clinical Director, Director of Research, Department of Radiation Oncology, New York Proton Center, 225 \\ East $126^{\text {th }}$ Street New York, NY 10035, USA. Email: ichoi@nyproton.com.
}

\begin{abstract}
Proton therapy (PT) has long been touted as a superior radiotherapy technology given its ability to stop dose at the distal end of the beam range, thereby better sparing normal tissues from unwanted irradiation dose compared with traditional photon therapy. While advantageous in theory, there is a limit to this superiority if the technology is not properly controlled and calibrated to account for a multitude of new factors that come into play when attempting to deliver radiation in such a precise manner. With a steady growth in proton centers being developed nationally and worldwide, it is of ever-increasing importance that the technology is delivered optimally. In the last several decades, proton technology has been refined, adjusted, and improved, advancing from passive scattering technology, to uniform scanning PT, to the most recent iteration of pencil beam scanning proton therapy (PBS-PT). Concomitantly, there have been adjunct advancements and technologies developed to support the continued refinement and precision of PT to allow for better targeting of increasingly complex tumors, including reduction in spot size, integration of volumetric on-board imaging (OBI), increased utilization of adaptive planning, improvements in motion management, development of optimization algorithms, and application of relative biologic effectiveness (RBE) and linear energy transfer (LET)-based planning, among others. These have allowed this ultra-precise form of radiotherapy to become ever-closer to reaching its full potential as the precision modality of choice in the field of radiation oncology for many disease presentations.
\end{abstract}

Keywords: Proton therapy (PT); precision medicine; radiation therapy; advanced technology; motion management

Received: 25 May 2020; Accepted: 22 October 2020; Published: 30 March 2021.

doi: $10.21037 /$ tro-20-42

View this article at: http://dx.doi.org/10.21037/tro-20-42

While the majority of radiation therapy courses are currently delivered with photon therapy, including 3 -dimensional conformal radiation therapy (3D-CRT) and intensity-modulated radiation therapy (IMRT), proton therapy (PT) has emerged as a way to reduce dose to normal tissues and potentially allow safer escalation of the biologically effective dose of treatment, delivery of trimodality therapy, and reirradiation treatments (1). Until the mid- to late-2000s, PT was a limited radiation modality only available at a handful of institutions in the United States (2). An earlier iteration of proton technology, passive scattering proton therapy (PS-PT), utilized the unique immediate stopping power of heavy charged particles to reduce or eliminate irradiation dose to tissues along the beam path beyond the intended target volume. However, PS-PT had several major inherent challenges, including imprecise dose deposition with scattered proton beams, inability to modulate or sculpt dose resulting in limited dose 


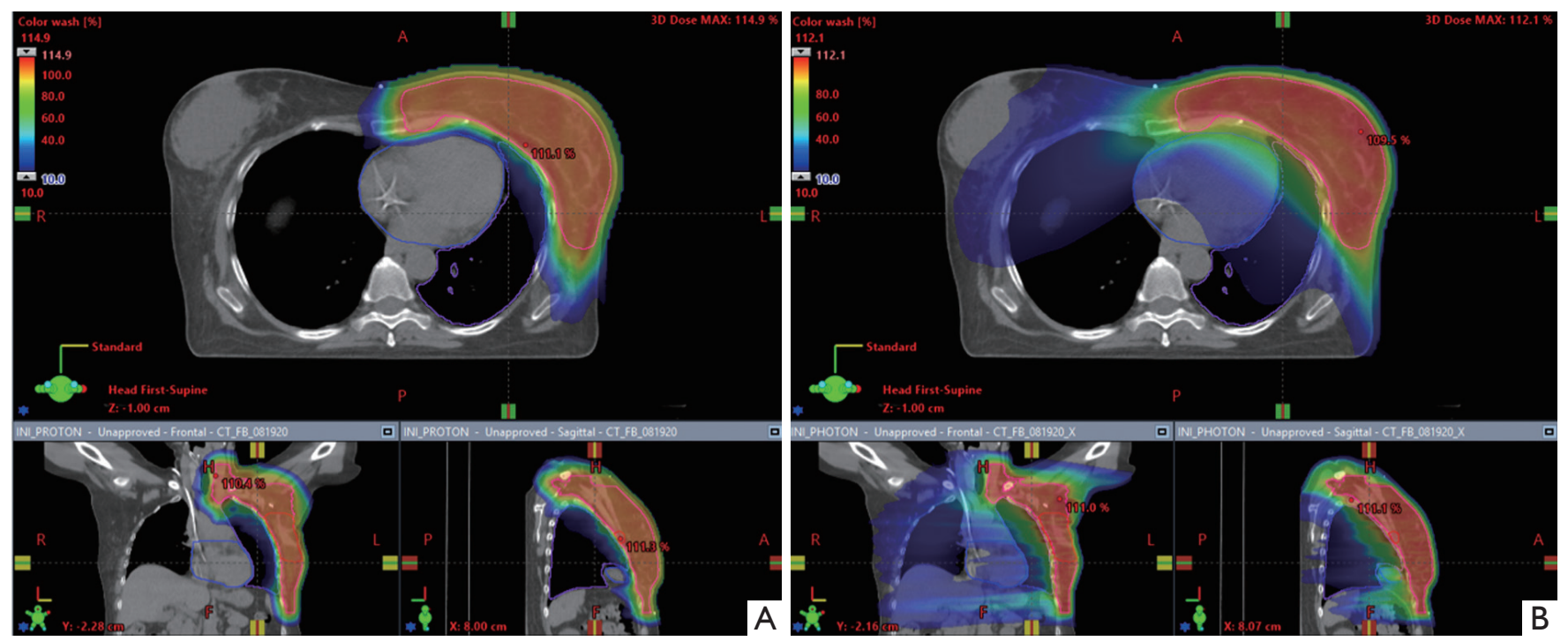

Figure 1 Comparison comprehensive breast cancer plans. Young woman with node-positive intraductal carcinoma of the left breast status post lumpectomy and axillary lymph node dissection and chemotherapy planned to receive whole breast and comprehensive nodal irradiation, inclusive of the left internal mammary nodes. Axial (top), coronal (left), sagittal (right) representative slices of comparative (A) proton therapy and (B) photon therapy plans. Proton therapy plan was designed with pencil beam scanning and photon therapy plan was designed with volumetric modulated arc therapy. The majority of heart receives some incidental unintended irradiation with photon therapy, whereas proton therapy optimizes tumor volume coverage while significantly reducing dose to the heart, ipsilateral and contralateral lungs, and liver. Dose gradient: $10 \%$ of the prescription dose (blue) to the dose maximum (red).

conformality, inadequate ability to calculate uncertainties due to heterogeneous tissue density, lack of technology to monitor intrafraction motion, and lack of volumetric on-board imaging (OBI) capabilities, among others. In the years to follow, these limitations would begin to be addressed, as the growing realization of the potentials of PT warranted continued refinement.

In the early days of PT, clinical application of the technology was most commonly for ocular tumors, chordomas, and the pediatric population, as the benefit of sparing still-developing tissues in children was irrefutable. To this day, the use in the pediatric population is still the most ubiquitous and uniformly accepted indication for PT. As improvements were made in proton technology, evolving first to uniform scanning proton therapy (US-PT) and then to the most modern iteration of pencil beam scanning proton therapy (PBS-PT) (Figure 1), the potential benefit of PT to minimize toxicities and better preserve quality of life across additional disease sites has become more apparent (3-7).

The advent of US-PT allowed for more precise proton delivery by enabling dose deposition in successive layers using scanning magnets, resulting in a more conformal dose distribution compared with that achievable with PS-
PT (8). However, US-PT is still limited to the use of only a single beam energy per layer, without the option of energy modulation during dose delivery of each layer. PBS-PT, representing the third generation of $\mathrm{PT}$, introduced the most significant advancement in PT to date. Layer-by-layer deposition of dose to individual spots in the target volume using a continuously modulated pencil beam enables dose painting and unparalleled precision and conformality of dose deposition to a customized target volume.

Additional improvements to address a number of earlier gaps in the technology were also developed during this time. Advances in OBI, spot size, motion management techniques, robust evaluation, and adaptive planning have pushed PT further forward, allowing it to grow ever-closer to harnessing its true potential through increased precision and accuracy (9-11). New complexities introduced by PBSPT were also unveiled, solutions to which have been and are still being refined, as will be further discussed.

With the continued improvements in PT and increasing recognition across the field that PT possesses the capability of benefitting a diverse group of patients, additional centers academic and private, hospital based and freestanding-have been developed across the United States, incorporating the 
newer iterations of PT and its supporting technologies. In fact, by August 2020, 35 centers are in operation nationally, whereas just 5 centers were in operation a decade earlier before 2010 (2).

\section{Spot size}

Optimal PBS-PT delivery is highly dependent on the specific parameters of the proton machine available at each center. Spot size has been found to be one of these integral beam characteristics that can determine if PBSPT technology results in superior, equivalent, or even worse dosimetry compared with PS-PT. Large spot sizes (variably defined as full width at half maximum for spot size at isocenter $0=6-15 \mathrm{~mm}$ ) may result in pencil beams with wider lateral profiles and more shallow penumbrae, leading to dose profiles that are no longer superior to those of PS-PT $(10,12,13)$. This is particularly true in the case of small targets, for which large spot sizes, especially in the periphery of the target volume, may lead to significant distal dose deposition and a wider penumbra, leading to higher doses to adjacent organs at risk (14). In addition, the use of smaller spot sizes (variably defined as full width at half maximum for spot size at isocenter $0=2-6 \mathrm{~mm}$ ) by definition requires employing a greater number of spots throughout the target volume. This allows for a greater degree of dose compensation for surrounding spots and/or planning inhomogeneities and results in a more homogeneous and accurate dose distribution.

PBS-PT delivery with small spot sizes have been shown in multiple institutional series to provide a significant clinical advantage compared with PS-PT, whereas PBSPT technology using larger spot sizes have not $(10,12)$. In specific clinical scenarios, larger spots sizes were once thought to be advantageous in providing a more robust dose distribution, particularly with moving tumors in the thorax, as larger spots were believed to be less susceptible to motion effects $(15,16)$. However, these studies were performed prior to the implementation of robust optimization (RO) in proton planning, and investigations using $\mathrm{RO}$ to account for motion and the interplay effect have since discounted this notion. In fact, to the contrary, as demonstrated in a planning study of lung cancer patients by Liu and colleagues, treatment plans using smaller spot sizes resulted in statistically significantly lower dose delivery to critical thoracic structures, including the heart and esophagus, compared with plans using larger spot sizes (13). This is likely due to the increased agility of dose deposition and compensation between small spots that is of particular importance for tumors subject to substantial motion, as higher levels of robustness can be achieved through modulation of spots to account for motion and the interplay effect, along with the availability of a finer brush for dosepainting more precisely in the tumor volume $(12,17,18)$.

Ideally, to account for a diversity of clinical scenarios, target volumes, and technical capabilities available, proton centers will have the flexibility to fluctuate between small and large spot sizes to maintain the agility needed to customize and optimize the treatment plan for each individual patient. As such, proton machine vendors have taken steps to develop the technology needed to create smaller spot sizes, and recent proton centers have taken this critical metric into account more consistently during the proton center development and commissioning process.

\section{Volumetric OBI}

PT is uniquely sensitive to changes in tissue density along its beam path. Any deviation along its beam path can result in significant dose perturbation and inaccurate treatment delivery of the proton beamlet, which delivers dose with submillimeter precision, resulting in significant range and setup uncertainties. As proton technology continues to increase in the precision with which it delivers its dose, in particular with the dose painting capability of PBS-PT and intensity-modulated proton therapy (IMPT), it is of growing importance to ensure target volume alignment mirrors planning setup and treatment plan design with millimeter precision to minimize any margin of error and avoid marginal misses and/or overdosing of adjacent critical structures.

To achieve this level of daily setup accuracy, image guidance with volumetric imaging is necessary, generally in the form of in-room cone beam CT (CBCT). Early proton centers were not designed with this type of OBI, and they instead relied on KV or MV portal imaging, limiting the array of disease sites that could be safely treated with this precision treatment modality. With the advent of PBS-PT, the need for more robust imaging to confirm accuracy of setup grew more apparent, and with increasing commercial availability, more modern proton centers now have this capacity built into the treatment machine (19). This allows for a planning margin on the order of only $3 \mathrm{~mm}$ to be included in the planning algorithm (robust or beamspecific PTV margin), significantly less than the 5-10 mm setup error and smearing distance incorporated for proton 

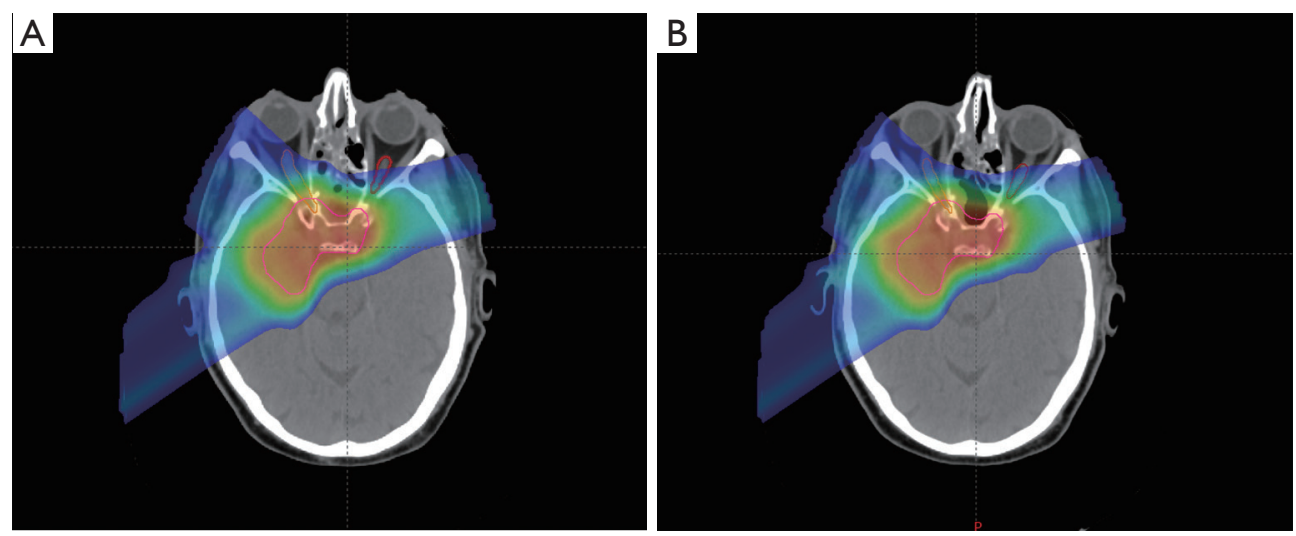

Figure 2 Base of skull adaptive plan. A patient with a chordoma of the right petrous apex status post debulking with gross residual disease. (A) Initial plan designed to treated with proton therapy to a planned dose of 78 Gy in 2 Gy daily fractions. (B) After 28 Gy, decreased density of the sinus fillings was evident. As a result, the right posterior oblique field dose end ranged further than originally planned, delivering more dose than desired to the left optic nerve. Continuing with this plan would exceed the left optic nerve tolerance, and an adaptive replan was required. Clinical Target Volume in pink, Right Optic Nerve in orange, Left Optic Nerve in red. Dose gradient: 10\% of the prescription dose (blue) to the dose maximum (red).

planning without CBCT or PBS-PS capability (20). This is particularly important since these larger planning margins diminish the potential tissue sparing that would otherwise be achievable with PT.

In-room volumetric imaging has also been a critical component of moving to treating more complex tumor sites, particularly those subject to internal inter- and intrafraction motion (11). Techniques to address respiratory motion (to be discussed in more detail below) and especially the delivery of stereotactic body PT largely require the support of in-room CT imaging in the form of CBCT or, less commonly, CT on rails, to sufficiently verify the internal anatomy and to confirm the treatment respiratory phase (in the case of deep inspiratory breath hold or respiratory gating) (21). Soft tissue visualization is also a critical component in the treatment of intra-abdominal tumors, which are subject to daily positional variation along with respiratory motion. For these tumors, target volume positioning in relation to surrounding gastrointestinal structures and motion management are critical to ensure precise dose delivery and to avoid undue toxicities to adjacent sensitive structures such as the bowel and kidneys. Finally, on-board CT imaging is necessary to assess for interfractional anatomical changes due to weight loss, fluid accumulation, gastrointestinal luminal density variability, inflammation, tumor change, and sinus/airway filling, which is critical given the sensitivity of proton particles to changes in density (11).

\section{Adaptive planning}

Due to the sensitivity of the proton beam to tissue density, any changes in external or internal anatomy can result in significant alterations in the planned proton dose distribution. Close monitoring throughout the course of radiation to assess for interfraction anatomical differences, which, as mentioned above, can arise due to a multitude of tumor- and patient-related factors (weight change, fluid fluctuation, tumor shrinkage/growth, differential air gap filling) is needed to ensure plan accuracy and the consistency of dose delivery for the duration of treatment (Figure 2). The value of on-treatment plan evaluation and adaptive planning with significant anatomical alteration has been demonstrated across multiple disease sites (22-26). Wu and colleagues found that in patients with oropharyngeal cancer, verification CT scan in the fourth week of treatment revealed significant reductions in CTV and parotid irradiation volumes of up to $12 \%$, along with significant under- and over-dosing of CTVs and OARs, respectively (22). In a study of patients with mobile non-small cell lung cancer, weekly 4DCT adaptive plans were created, demonstrating a mean increase in the maximum dose to the spinal cord of 4.4 Gy and an average $4 \%$ increase in contralateral lung receiving at least 5 Gy (23).

Implementation of adaptive planning in a meaningful way requires (I) the ability to recognize the need for replanning followed by (II) a workflow to develop, evaluate, and start modified plans quickly and accurately. This process 
remains a challenge in clinical practice. Ongoing questions under investigation to optimize adaptive replanning include uncertainty regarding the optimal method of incorporating dose contribution from the adaptive plan to the overall plan, ideal methods of accurate and efficient OAR and target volume modification utilizing rigid or deformable image registration, and the ability to perform reliable dose calculations from CBCT versus the need for de novo CT simulation images. Attempts to make these processes more efficient and reliable have been at the forefront of the industry's technological innovation as the field becomes increasingly aware of how critical these tools are to ensure that the precision achievable with PT is not diminished by a lack of supporting technological and clinical infrastructure.

\section{Motion management}

Early uses of PT largely involved treatment of static disease sites with minimal to no concern for intrafraction motion, such as for the treatment of CNS tumors in pediatric, and later, adult patients, in which a custom mask was used for immobilization, and prostate cancer, for which a rectal balloon was commonly used to mitigate intra- and interfraction variability. With the gradual expansion of potential indications for PT, along with increased awareness of the susceptibility of the proton beams to density changes in the beam path, yet a new layer of uncertainty was uncovered that could perturb dose delivery and degrade plan robustness: intrafraction motion. This is most commonly due to patient respiratory motion, most pronounced in tumors of the thorax and upper abdomen (27).

With the introduction of PBS-PT, and particularly the advent of multi-field optimized PBS-PT, the potential for degradation of plan robustness due to respiratory motion is particularly pronounced due to the interplay effect (28-31), a phenomenon not as significant in the static field delivery of PS-PT (32) or with single-field optimized PBS-PT $(21,33)$. In photon therapy, motion management has become increasingly important as the field continues to move towards more ubiquitous adoption of hypofractionated treatment schedules and stereotactic procedures, which are dependent on high levels of setup accuracy and minimal intrafraction motion to deliver these high doses per fraction (34). Multiple techniques for motion management and evaluation have been implemented including active breathing control, respiratory gating, abdominal compression, and deep inspiratory breath hold $(31,35)$. These same techniques are now being increasingly applied to the delivery of PT as the technology is modified for compatible use with existing proton technology.

Additionally, the use of 4DCT for treatment planning is a key technique to assess the extent of motion and subsequent need for more interventions to ensure robust treatment setup and delivery. Worst-case (or second-worstcase) scenario plan optimization may also allow for the incorporation of known changes with respiratory motion and the development a more robust treatment plan (36). Another potential approach to mitigate dose degradation and improve dose homogeneity due to motion includes using larger spot sizes, particularly without the ability to perform robust planning analyses (31). Thus, the flexibility of having multiple spot sizes available at an institution for use in different scenarios is beneficial, as mentioned above. Continued study in identifying optimal approaches to account for motion management in PT delivery to allow for robust and precise dose delivery will be needed as treatment of tumors most subject to respiratory motion becomes more common.

\section{Robust optimization (RO)}

Finally, the treatment planning systems for PT continue to reach new levels of sophistication, incorporating algorithms that can account for uncertainties introduced when delivering the ultraprecise proton beam with IMPT. Setup and range uncertainties in a variety of forms can cause a significant degradation of planned proton dose distributions, even with a slight deviation from the nominal plan. The result of such error can potentially lead to overdosing adjacent critical structures and/or underdosing target volumes $(9,37,38)$. This phenomenon is especially important to consider in the case of multi-field optimized PBS-PT planning, in which the inhomogeneity of spot intensity is compensated by spots deposited from beamlets entering from different directions. With any level of uncertainty, the tenuous balance of these spot contributions could be perturbed, again leading to over- or under-dosing around and within the target volume (9). Using RO in the treatment planning process introduces a technique that can predict and account for these critical uncertainties by evaluating all uncertainty scenarios simultaneously, allowing treatment plans to be optimized based on these possible deviations in the planned treatment delivery.

Several different approaches to RO have been introduced, including worst-case optimization (39-41), probabilistic and linear programming to identify idealized 
2-dimensional geometry for range uncertainties (42), and minimax optimization $(43,44)$. RO has been integrated into many modern treatment planning systems and has now largely become an industry standard for more accurate and safe proton treatment delivery. Continued investigations are underway to increase the speed of these optimization calculations and to identify the most efficient and efficacious $\mathrm{RO}$ algorithm to improve the integration of $\mathrm{RO}$ into the clinical workflow (45).

\section{Relative biologic effectiveness (RBE) treatment planning}

Beyond algorithmic RO, proton beam arrangements and plans should be optimized to account for differences in RBE and linear energy transfer (LET) between protons and photons. PT dose is expressed in units of Gray $[G y(R B E)]$, the effective dose being the physical dose in Gray multiplied by RBE. Furthermore, the RBE is the ratio of dose of high-energy photons relative to dose of protons needed to produce the same biologic response. Protons generally average $10 \%$ greater biologic potency than photons, for an RBE of 1.1 (46). However, the RBE can significantly increase at the distal end of the Bragg peak (47). This has been reported to lead to excess toxicity when multiple beams have end ranged into a critical structure immediately distal to the target volume $(48,49)$. Just as plans should be arranged to avoid having an increased RBE in a critical structure, there is a potential to use this increase effectiveness to improve tumor control, especially for hypoxic and/or radioresistant tumors. While commercially available treatment planning systems currently do not allow treatment plans to be modified based on biological considerations, investigation into the potential role of $\mathrm{PT}$ to overcome radioresistance through its high $\mathrm{RBE}$ at the end of the Bragg Peak is warranted. As such, RBE and/or LET optimized plans hold the potential to reduce normal tissue complications and potentially enhance tumor control.

\section{Secondary malignancies}

By significantly reducing the integral dose and normal tissues exposed to unnecessary irradiation, PT has the potential to reduce the risk of late radiation-induced secondary malignancies compared with photon therapy. Modeling studies have predicted such secondary cancer reductions with protons across multiple disease sites, including the abdomen and pelvis (50) and the thorax (51).
This predicted secondary malignancy risk reduction, however, needs to be evaluated relative to the potential risks of neutron contamination from PT. Second cancers from neutron dose is thought to be significantly less than the direct risk reduction benefit from protons reducing integral dose, and this neutron dose is further reduced with PBS compared with passive scattering PT (50,52).

The available clinical data support these models and have demonstrated that PT reduces the risk of secondary malignancies relative to photon therapy. In matching patients treated at the Harvard Cyclotron with patients treated with photons in the SEER registry, PT resulted in approximately half the rate of secondary malignancies (hazard ration $0.52, \mathrm{P}=0.009$ ) (53). More recently, in a 450,373 patient National Cancer Database analysis, PT led to only a third as many second cancers as IMRT (HR 0.31, $\mathrm{P}<0.0001)(54)$.

\section{Conclusions}

Radiation oncology is in the midst of a renaissance. PT provides clinicians with the ability to deliver radiotherapy with a level of precision and normal tissue sparing that are unprecedented and unparalleled by any prior iterations of external beam radiation technology. Significant advances in PT over the past two decades have further optimized this advanced treatment modality, furthering its potential applications to an increasingly diverse population of patients. The third generation of proton technology, PBS-PT, is the most recent iteration of PT and has opened the possibility for ultra-precise proton beam delivery. Ancillary technologies have been developed to support the complexities, unique characteristics, and tremendous precision of PT to provide practitioners with the tools to best harness the potentials of PT and optimize the therapeutic ratio for the modality. While the dosimetric advantages of PT continue to be undeniable, the translation of this benefit into clinically apparent toxicity, quality of life, and disease control benefits over photon therapy are still under study across many disease sites, and further study will be critical to advancing our understanding of how this technology will best be applied and integrated into the field in the future.

Even further advances in PT are expected as additionally investigations technologies develop and/or mature. As detailed above, RBE-based planning and LET painting may allow for improved tumor control and reduced toxicities. While MRI-based linear accelerators are increasingly available, magnetic resonance image-guided PT has yet 
to be realized and has the potential to better delineate and target tumors and also reduce toxicities. Finally, in vivo data of ultra-high dose rate FLASH treatment have demonstrated enhanced normal tissue protection (55). As powerful synchrotrons and cyclotrons may be optimal ways to deliver FLASH dose rates, there is great interested in FLASH delivered with PT as a future way to further optimize this advanced precision technology (56).

\section{Acknowledgments}

Funding: Supported by National Institutes of Health/ National Cancer Institute Memorial Sloan Kettering Cancer Center Support Grant/Core Grant No. P30CA008748, period: 1/1/19 - 12/31/23.

\section{Footnote}

Provenance and Peer Review: This article was commissioned by the Guest Editors (Chen-Hsi Hsieh, Hsin-Hua Nien, and Yu-Ming Huang) for the series "Precision Medicine for Cancer" published in Therapeutic Radiology and Oncology. The article has undergone external peer review.

Conflicts of Interest: All authors have completed the ICMJE uniform disclosure form (available at http://dx.doi. org/10.21037/tro-20-42). The series "Precision Medicine for Cancer" was commissioned by the editorial office without any funding or sponsorship. CBS serves as an unpaid editorial board member of Therapeutic Radiology and Oncology. Dr. CBS reports other from Varian Medical Systems, outside the submitted work. The authors have no other conflicts of interest to declare.

Ethical Statement: The authors are accountable for all aspects of the work in ensuring that questions related to the accuracy or integrity of any part of the work are appropriately investigated and resolved.

Open Access Statement: This is an Open Access article distributed in accordance with the Creative Commons Attribution-NonCommercial-NoDerivs 4.0 International License (CC BY-NC-ND 4.0), which permits the noncommercial replication and distribution of the article with the strict proviso that no changes or edits are made and the original work is properly cited (including links to both the formal publication through the relevant DOI and the license). See: https://creativecommons.org/licenses/by-nc-nd/4.0/.

\section{References}

1. Simone CB 2nd, Rengan R. The use of proton therapy in the treatment of lung cancers. Cancer J 2014;20:427-32.

2. Particle therapy facilities in clinical operation. Particle Therapy Co-Operative Group. Available online: https:// www.ptcog.ch/index.php/facilities-in-operation. Accessed September 14, 2020.

3. DeLaney TF, Kooy HM. Proton and Charged Particle Radiotherapy. Wolters Kluwer Health, 2015.

4. Paganetti H. Proton Therapy Physics. Taylor \& Francis, 2011.

5. Zheng Y, Ramirez E, Mascia A, et al. Commissioning of output factors for uniform scanning proton beams. Med Phys 2011;38:2299-306.

6. Baumann BC, Mitra N, Harton JG, et al. Comparative Effectiveness of Proton vs Photon Therapy as Part of Concurrent Chemoradiotherapy for Locally Advanced Cancer. JAMA Oncol 2020;6:237-46.

7. Verma V, Simone CB 2nd, Mishra MV. Quality of Life and Patient-Reported Outcomes Following Proton Radiation Therapy: A Systematic Review. J Natl Cancer Inst 2018;110.

8. Zheng Y. Adaptive Radiotherapy for Lung Cancer Using Uniform Scanning Proton Beams. In: Radiotherapy. Onal C. Editor. IntechOpen, 2017. http://dx.doi. org/10.5772.67445.

9. Lomax AJ. Intensity modulated proton therapy and its sensitivity to treatment uncertainties 1: the potential effects of calculational uncertainties. Phys Med Biol 2008;53:1027-42.

10. Kraan AC, Depauw N, Clasie B, et al. Impact of spot size variations on dose in scanned proton beam therapy. Phys Med 2019;57:58-64.

11. Veiga C, Janssens G, Teng CL, et al. First Clinical Investigation of Cone Beam Computed Tomography and Deformable Registration for Adaptive Proton Therapy for Lung Cancer. Int J Radiat Oncol Biol Phys 2016;95:549-59.

12. Moteabbed M, Yock TI, Depauw N, et al. Impact of Spot Size and Beam-Shaping Devices on the Treatment Plan Quality for Pencil Beam Scanning Proton Therapy. Int J Radiat Oncol Biol Phys 2016;95:190-8.

13. Liu C, Schild SE, Chang JY, et al. Impact of Spot Size and Spacing on the Quality of Robustly Optimized Intensity Modulated Proton Therapy Plans for Lung Cancer. Int J Radiat Oncol Biol Phys 2018;101:479-89.

14. Kralik JC, Xi L, Solberg TD, et al. Comparing proton treatment plans of pediatric brain tumors in two pencil 
beam scanning nozzles with different spot sizes. J Appl Clin Med Phys 2015;16:41-50.

15. Dowdell S, Grassberger C, Sharp GC, et al. Interplay effects in proton scanning for lung: a 4D Monte Carlo study assessing the impact of tumor and beam delivery parameters. Phys Med Biol 2013;58:4137-56.

16. Grassberger C, Dowdell S, Lomax A, et al. Motion interplay as a function of patient parameters and spot size in spot scanning proton therapy for lung cancer. Int J Radiat Oncol Biol Phys 2013;86:380-6.

17. Grosshans DR, Mohan R, Gondi V, et al. The role of image-guided intensity modulated proton therapy in glioma. Neuro Oncol 2017;19:ii30-ii37.

18. Kang M, Huang S, Solberg TD, et al. A study of the beamspecific interplay effect in proton pencil beam scanning delivery in lung cancer. Acta Oncol 2017;56:531-40.

19. Mohan R, Das IJ, Ling CC. Empowering Intensity Modulated Proton Therapy Through Physics and Technology: An Overview. Int J Radiat Oncol Biol Phys 2017;99:304-16.

20. Park PC, Cheung JP, Zhu XR, et al. Statistical assessment of proton treatment plans under setup and range uncertainties. Int J Radiat Oncol Biol Phys 2013;86:1007-13.

21. Chang JY, Zhang X, Knopf A, et al. Consensus Guidelines for Implementing Pencil-Beam Scanning Proton Therapy for Thoracic Malignancies on Behalf of the PTCOG Thoracic and Lymphoma Subcommittee. Int J Radiat Oncol Biol Phys 2017;99:41-50.

22. Wu RY, Liu AY, Sio TT, et al. Intensity-Modulated Proton Therapy Adaptive Planning for Patients with Oropharyngeal Cancer. Int J Part Ther 2017;4:26-34.

23. Hui Z, Zhang X, Starkschall G, et al. Effects of Interfractional Motion and Anatomic Changes on Proton Therapy Dose Distribution in Lung Cancer. Int J Radiat Oncol Biol Phys 2008;72:1385-95.

24. Kraan AC, van de Water S, Teguh DN, et al. Dose uncertainties in IMPT for oropharyngeal cancer in the presence of anatomical, range, and setup errors. Int J Radiat Oncol Biol Phys 2013;87:888-96.

25. Góra J, Kuess P, Stock M, et al. ART for head and neck patients: On the difference between VMAT and IMPT. Acta Oncol 2015;54:1166-74.

26. Simone CB 2nd, Ly D, Dan TD, et al. Comparison of intensity-modulated radiotherapy, adaptive radiotherapy, proton radiotherapy, and adaptive proton radiotherapy for treatment of locally advanced head and neck cancer. Radiother Oncol 2011;101:376-82.

27. Lin L, Souris K, Kang M, et al. Evaluation of motion mitigation using abdominal compression in the clinical implementation of pencil beam scanning proton therapy of liver tumors. Med Phys 2017;44:703-12.

28. Knopf AC, Hong TS, Lomax A. Scanned proton radiotherapy for mobile targets-the effectiveness of rescanning in the context of different treatment planning approaches and for different motion characteristics. Phys Med Biol 2011;56:7257-71.

29. Bert C, Grözinger SO, Rietzel E. Quantification of interplay effects of scanned particle beams and moving targets. Phys Med Biol 2008;53:2253-65.

30. Kraus KM, Heath E, Oelfke U. Dosimetric consequences of tumour motion due to respiration for a scanned proton beam. Phys Med Biol 2011;56:6563-81.

31. Grassberger C, Dowdell S, Sharp G, et al. Motion mitigation for lung cancer patients treated with active scanning proton therapy. Med Phys 2015;42:2462-9.

32. Kang Y, Zhang X, Chang JY, et al. 4D Proton treatment planning strategy for mobile lung tumors. Int J Radiat Oncol Biol Phys 2007;67:906-14.

33. Dowdell S, Grassberger C, Sharp G, et al. Fractionated Lung IMPT Treatments: Sensitivity to Setup Uncertainties and Motion Effects Based on Single-Field Homogeneity. Technol Cancer Res Treat 2016;15:689-96.

34. Molitoris JK, Diwanji T, Snider JW 3rd, et al. Optimizing immobilization, margins, and imaging for lung stereotactic body radiation therapy. Transl Lung Cancer Res 2019;8:24-31.

35. Boda-Heggemann J, Knopf AC, Simeonova-Chergou A, et al. Deep inspiration breast hold-based radiation therapy: A clinical review. Int J Radiat Oncol Biol Phys 2016;94:478-92.

36. Liu W, Liau Z, Schild SE, et al. Impact of respiratory motion on worst-case scenario optimized intensity modulated proton therapy for lung cancers. Pract Radiat Oncol 2015;5:e77-86.

37. Graeff C, Durante M, Bert C. Motion mitigation in intensity modulated particle therapy by internal target volumes covering range changes. Med Phys 2012;39:6004-13.

38. Lowe M, Aitkenhead A, Albertini F, et al. A robust optimization approach accounting for the effect of fractionation on setup uncertainties. Phys Med Biol 2017;62:8178-96.

39. Pflugfelder D, Wilkens JJ, Oelfke U. Worst case optimization: a method to account for uncertainties in the optimization of intensity modulated proton therapy. Phys Med Biol 2008;53:1689-700. 
40. Liu W, Zhang X, Li Y, et al. Robust optimization in intensity-modulated proton therapy. Med Phys 2012;39:1079-91.

41. Yang Z, Li H, Li Y, et al. Statistical evaluation of worstcase robust optimization intensity-modulated proton therapy plans using an exhaustive sampling approach. Radiat Oncol 2019;14:129.

42. Unkelbach J, Bortfeld T, Martin BC, et al. Reducing the sensitivity of IMPT treatment plans to setup errors and range uncertainties via probabilistic treatment planning. Med Phys 2009;36:149-63.

43. Fredriksson A, Forsgren A, Hårdemark B. Minimax optimization for handling range and setup uncertainties in proton therapy. Med Phys 2011;38:1672-84.

44. Stuschke M, Kaiser A, Pottgen C, et al. Potentials of robust intensity modulated scanning proton plans for locally advanced lung cancer in comparison to intensity modulated photon plans. Radiother Oncol 2012;104:45-51.

45. Li Y, Niemela P, Liao L, et al. Selective robust optimization: a new intensity-modulated proton therapy optimization strategy. Med Phys 2015;42:4840-7.

46. Paganetti H, Niemierko A, Ancukiewicz M, et al. Relative biological effectiveness (RBE) values for proton beam therapy. Int J Radiat Oncol Biol Phys 2002;53:407-21.

47. Chaudhary P, Marshall TI, Perozziello FM, et al. Relative biological effectiveness variation along monoenergetic and modulated Bragg peaks of a $62-\mathrm{MeV}$ therapeutic proton beam: a preclinical assessment. Int J Radiat Oncol Biol Phys 2014;90:27-35.

48. Kralik SF, Ho CY, Finke W, et al. Radiation Necrosis in Pediatric Patients with Brain Tumors Treated with Proton Radiotherapy. AJNR Am J Neuroradiol 2015;36:1572-8.

doi: $10.21037 /$ tro-20-42

Cite this article as: Choi JI, Hasan S, Press RH, Chhabra AM, Simone CB 2nd. Sharpening the particle knife: the evolution of ultra-precise proton therapy. Ther Radiol Oncol 2021;5:3.
49. McDonald MW, Linton OR, Calley CS. Dose-volume relationships associated with temporal lobe radiation necrosis after skull base proton beam therapy. Int J Radiat Oncol Biol Phys 2015;91:261-7.

50. Simone CB 2nd, Kramer K, O'Meara WP, et al. Predicted rates of secondary malignancies from proton versus photon radiation therapy for stage I seminoma. Int J Radiat Oncol Biol Phys 2012;82:242-9.

51. Vogel J, Lin L, Litzky LA, et al. Predicted Rate of Secondary Malignancies Following Adjuvant Proton Versus Photon Radiation Therapy for Thymoma. Int J Radiat Oncol Biol Phys 2017;99:427-33.

52. Zacharatou Jarlskog C, Paganetti H. Risk of developing second cancer from neutron dose in proton therapy as function of field characteristics, organ, and patient age. Int J Radiat Oncol Biol Phys 2008;72:228-35.

53. Chung CS, Yock TI, Nelson K, et al. Incidence of second malignancies among patients treated with proton versus photon radiation. Int J Radiat Oncol Biol Phys 2013;87:46-52.

54. Xiang M, Chang DT, Pollom EL. Second cancer risk after primary cancer treatment with three-dimensional conformal, intensity-modulated, or proton beam radiation therapy. Cancer 2020;126:3560-8.

55. Favaudon V, Caplier L, Monceau V, et al. Ultrahigh doserate FLASH irradiation increases the differential response between normal and tumor tissue in mice. Sci Transl Med 2014;6:245ra93.

56. Griffin RJ, Limoli CL, Simone CB. Radiation Research Special Issue: New Beam Delivery Modalities are Shaping the Future of Radiotherapy. Radiat Res 2020;194:567-70. 